\title{
Colangite biliar primária: um relato de caso
}

\author{
Primary biliary cholangitis: a case report \\ Colangitis biliar primaria: reporte de caso
}

Liliana Sampaio Costa Mendes ${ }^{1 *}$, Caio Teles Batista ${ }^{2}$, Gabriela Muniz Carneiro ${ }^{3}$, Kauê de Mello Aleixo ${ }^{4}$, Wladimir Magalhães Freitas ${ }^{5}$.

\section{RESUMO}

Objetivo: Elucidar aspectos diagnósticos e terapêuticos acerca da Colangite Biliar Primária (CBP), doença rara e progressiva que requer identificação precoce a fim de evitar suas complicações. Detalhamento do caso: Paciente feminino, 55 anos, procurou atendimento por alterações de enzimas hepáticas. Negava prurido, astenia e insônia. Exame físico normal. Solicitados exames laboratoriais: TGO 83 U/L; TGP 118 U/L; FA 277 U/L; GGT 310 U/L; AMA 1:40; ANTILKM negativo; AAML negativo; FAN 1:160. Biópsia hepática: infiltrado portal focal e discreto, sinusoidal moderado infiltrado linfoplasmocitário em ductos biliares septais com células inflamatórias. Foi realizado tratamento para CBP com ácido ursodesoxicólico $15 \mathrm{mg} / \mathrm{kg}$. Retorna assintomática e com melhora do quadro laboratorial. Considerações finais: A Colangite Biliar Primária é caracterizada por uma sintomatologia variada, o que dificulta o diagnóstico precoce e início do tratamento. Desse modo, se faz necessário maior conhecimento a respeito da doença pela comunidade médica, com propósito de impedir sua progressão e complicações.

Palavras-chave: Colangite biliar primária, Cirrose biliar primária, Cirrose biliar.

\begin{abstract}
Objective: To elucidate diagnostic and therapeutic aspects about Primary Biliary Cholangitis (PBC), a rare and progressive disease that requires early identification in order to avoid complications. Details of the case: Female patient, 55 years old, sought care for changes in liver enzymes. Denied Pruritus, asthenia and insomnia. Normal physical examination. Requested laboratory tests: TGO 83 U / L; TGP 118 U / L; FA 277 U / L; GGT 310 U / L; AMA 1:40; Negative ANTILKM; Negative AAML; FAN 1: 160. Hepatic biopsy: focal and discrete portal infiltrate, moderate sinusoidal lymphoplasmacytic infiltrate in septal bile ducts with inflammatory cells. Treatment for PBC) was performed with ursodeoxycholic acid $15 \mathrm{mg} / \mathrm{kg}$. Patient returns asymptomatic and with improvement of the laboratory tests. Final considerations: Primary Biliary Cholangitis is characterized by a variety of symptoms, which makes it difficult to diagnose early and start treatment. Thus, it is necessary to know more about the disease by the medical community, in order to prevent its progression and complications.
\end{abstract}

Keywords: Primary biliary cholangitis, Primary biliary cirrhosis, Biliary cirrhosis.

\section{RESUMEN}

Objetivo: Dilucidar los aspectos diagnósticos y terapéuticos de la Colangitis Biliar Primaria (CBP), una enfermedad rara y progresiva que requiere una identificación precoz para evitar sus complicaciones. Detalles del caso: Paciente de sexo femenino, 55 años, solicitó atención por alteraciones de las enzimas hepáticas. Negó prurito, astenia e insomnio. Examen físico normal. Pruebas de laboratorio solicitadas: TGO 83 U / L; TGP 118 U / L; FA 277 U / L; GGT 310 U / L; AMA 1:40; ANTILKM negativo; AAML negativo; VENTILADOR 1: 160. Biopsia hepática: infiltrado portal focal y discreto, infiltrado linfoplasmocitario sinusoidal moderado en conductos biliares septales con células inflamatorias. El tratamiento para la CBP se realizó con ácido ursodesoxicólico $15 \mathrm{mg} / \mathrm{kg}$. Vuelve asintomático y con mejoría del cuadro de laboratorio. Consideraciones finales: la colangitis biliar primaria se caracteriza por una variedad de síntomas, lo que dificulta el diagnóstico temprano y el inicio del tratamiento. Por ello, es necesario conocer más sobre la enfermedad por parte de la comunidad médica, con el fin de prevenir su progresión y complicaciones.

Palabras clave: Colangitis biliar primaria, Cirrosis biliar primaria, Cirrosis biliar.

\footnotetext{
${ }^{1}$ Hospital de Base, Brasília - DF. *E-mail: mendesliliana2@gmail.com

2 Centro Universitário do Planalto Central Apparecido dos Santos (UNICEPLAC), Gama - DF.

${ }^{3}$ Centro Universitário de Brasilia (UniCEUB), Brasília - DF.

4 Escola Superior de Ciências da Saúde (ESCS), Brasília - DF

5 Instituto Biocárdios, Brasília - DF.
} 


\section{INTRODUÇÃO}

A colangite biliar primária (CBP) é considerada uma doença crônica, autoimune e de caráter progressivo, que cursa com inflamação e destruição crônica hepato-biliar. Possui assinatura sorológica característica, o anticorpo antimitocondrial (AMA) (BEUERS U, et al., 2015). Acredita-se que a etiologia da CBP seja devido a uma combinação de fatores de risco genéticos e gatilhos ambientais (SELMI C, et al., 2011).

A prevalência geral da doença clínica em várias populações tem sido difícil de estimar devido à raridade da doença. As estimativas variam entre 19 e 402 casos de CBP por milhão de habitantes (SELMI C, et al., 2011). Ocorre predomínio na população feminina, geralmente em maiores de 40 anos de idade (BEUERS U, et al., 2015).

O quadro clínico mais comum é a forma assintomática, prevalente em até $50 \%$ a $60 \%$ dos casos. Isso faz com que o diagnóstico ocorra principalmente a partir de achados laboratoriais. Quando sintomática, a apresentação de fadiga e prurido são as mais comuns. Portanto, inicialmente pode apresentar-se de forma bastante inespecífica (PANDIT S, et al. 2021). A epidemiologia e quadro clínico inicial justificam o contato inabitual e a maior probabilidade de atraso no diagnóstico, podendo acarretar em prejuízos ao paciente e que poderiam ser sanados a partir da correta conduta investigativa (SELMI C, et al., 2011).

O diagnóstico é realizado através da tríade: (1) elevações das enzimas canaliculares (FA e GTT) pelo menos 1,5 vezes o limite superior normal; (2) presença de autoanticorpos (AMA) em um título de 1:40 ou superior (ou outros autoanticorpos específicos de CBP [sp100 ou gp210] se AMA for negativo) e (3) evidência histopatológica de colangite crônica não supurativa e destrutiva. O diagnóstico pode ser presumível na presença de dois dos três critérios (KUMAGI T e HEATHCOTE E, et al., 2008). Embora a biópsia hepática geralmente não seja recomendada para fazer o diagnóstico, ela fornece informações úteis para a avaliação e estadiamento da doença (SELMI C, et al., 2011).

O tratamento universalmente aceito para os casos de CBP é o uso do ácido ursodesoxicólico (UDCA), droga segura e bem tolerada pelos pacientes. Na ausência da terapêutica adequada, os portadores de CBP tendem a evoluir progressivamente para insuficiência hepática, cirrose, e consequentemente necessidade de transplante hepático. Foi demonstrado que o uso do UDCA proporciona melhora na sobrevida livre de transplante hepático, tanto em pacientes com doença em fase inicial, quanto naqueles de evolução avançada, reforçando a importância do diagnóstico e terapia adequada (HARMS MH, et al., 2019).

O objetivo deste trabalho é elucidar aspectos diagnósticos e terapêuticos de uma doença rara, com base em um relato de caso.

\section{DETALHAMENTO DO CASO}

Este relato de experiência seguiu as recomendações previstas pela Resolução 466/12 do Conselho Nacional de Saúde do Ministério da Saúde atendendo às exigências éticas e científicas fundamentais. O projeto foi submetido à Plataforma Brasil para avaliação de um Comitê de Ética em Pesquisa com Seres Humanos e foi aprovado sob o parecer № 4.517.206. O Termo de Consentimento Livre e Esclarecido foi assinado pela paciente da pesquisa, permitindo a utilização exclusiva de dados colhidos em prontuário eletrônico, sendo garantido e mantido extremo sigilo em relação aos dados identificadores.

Paciente, sexo feminino, 55 anos, natural e procedente do Distrito Federal (DF) procurou atendimento em serviço especializado em Hepatologia devido à persistência de alterações das enzimas hepáticas em exames de rotina. Relatou que, há 5 anos, buscou investigar as alterações enzimáticas, recebendo diagnóstico de Lesão Hepática Induzida Por Fármacos (LHIF ou DILI), com alta.

$\mathrm{Na}$ consulta, informou que irmã, tia e avó possuem colangite de origem genética. Negou prurido, astenia e insônia. Referiu dislipidemia, em uso de rosuvastatina $5 \mathrm{mg}$ há 4 anos. Negou Diabetes Melitus (DM), Hipertensão Arterial Sistêmica (HAS), tireoidopatia, hemotransfusões, tabagismo, drogas e passou a evitar o uso de analgésicos. Relatava acompanhamento com médico ortomolecular há 9 anos, fazendo uso de fórmulas: Pancreatina, Vanádio, Glicosamina, Co-Q10, Dehidroepiandrosterona (DHEA), Manaquinina, Boro, vitamina $\mathrm{D}$ e Cranberry. Relatou a suspensão do uso de fórmulas, Sertralina e Nicotinamida há 3 meses da primeira consulta. 
Paciente apresentava bom estado geral, lúcida, orientada, afebril, corada, anictérica, hidratada, $59 \mathrm{~kg}$, $1,60 \mathrm{~m}$, pressão arterial de 130/80 mmHg, frequência cardíaca 75 bpm e saturação de oxigênio $97 \%$. Exame físico cardiopulmonar sem alterações. Abdômen apresentava configuração normal, sem circulação colateral visível, sem sinais de ascite e ausência hepatoesplenomegalia. Ruídos hidroaéreos normais. Não se observavam telangiectasias, xantomas, eritema palmar ou outros sinais de hepatopatia crônica.

Foram solicitados exames laboratoriais: Transaminase Glutâmico-Oxalacética (TGO) $83 \mathrm{UI} / \mathrm{mL}$ (valor de referência $34 \mathrm{UI} / \mathrm{ml}$ ); Transaminase Glutâmico-Pirúvico (TGP) $118 \mathrm{UI} / \mathrm{mL}$ (valor de referência $49 \mathrm{UI} / \mathrm{ml}$ ); Fosfatase Alcalina (FA) $277 \mathrm{UI} / \mathrm{ml}$ (valor de referência $116 \mathrm{UI} / \mathrm{ml}$ ); Gama Glutamil Transferase (GGT) 310 $\mathrm{Ul} / \mathrm{ml}$ (valor de referência $38 \mathrm{UI} / \mathrm{ml}$ ); Anticorpos Anti-Mitocôndria (AMA) 1:40; Anticorpos ANTI-LKM1 negativo; Anticorpo Anti-Músculo liso (AAML) negativo; Fator Antinuclear (FAN) 1:160 citoplasmático reticulado; Glicose-6-Fosfato Desidrogenase (G6PD) $7.2 \mathrm{U} / \mathrm{g}$; Proteína alfa-1-antitripsina (A1AT) $139 \mathrm{mg} / \mathrm{dL}$; Colesterol $242 \mathrm{mg} / \mathrm{dL}$; Triglicerídeos $53 \mathrm{mg} / \mathrm{dL}$; Lipoproteínas de baixa densidade (LDL) 159 mg/dL; Lipoproteínas de alta densidade (HDL) $70 \mathrm{mg} / \mathrm{dL}$. Demais exames laboratoriais sem alterações

Foi realizado biópsia hepática percutânea que demonstrou infiltrado linfoplasmocitário portal focal e discreto, sinusoidal moderado (linfócitos, eosinófilos e ocasionais plasmócitos); infiltrado linfoplasmocitário em ductos biliares septais; observou-se ausência de rarefação de ductos em tríades portais e placa limitante íntegra; CD68+ em células de kupffer e no infiltrado inflamatório portal e sinusoidal; CD 45+ células inflamatórias sinusoidais, portais e intrabiliares septais; CD138+ ocasional células inflamatórias portais e intrabiliares septais. Estas alterações são sugestivas de cirrose biliar primária no estádio 1, de acordo com a classificação de Peter Scheuer (Figura 1).

Figura 1 - Biópsia hepatica.
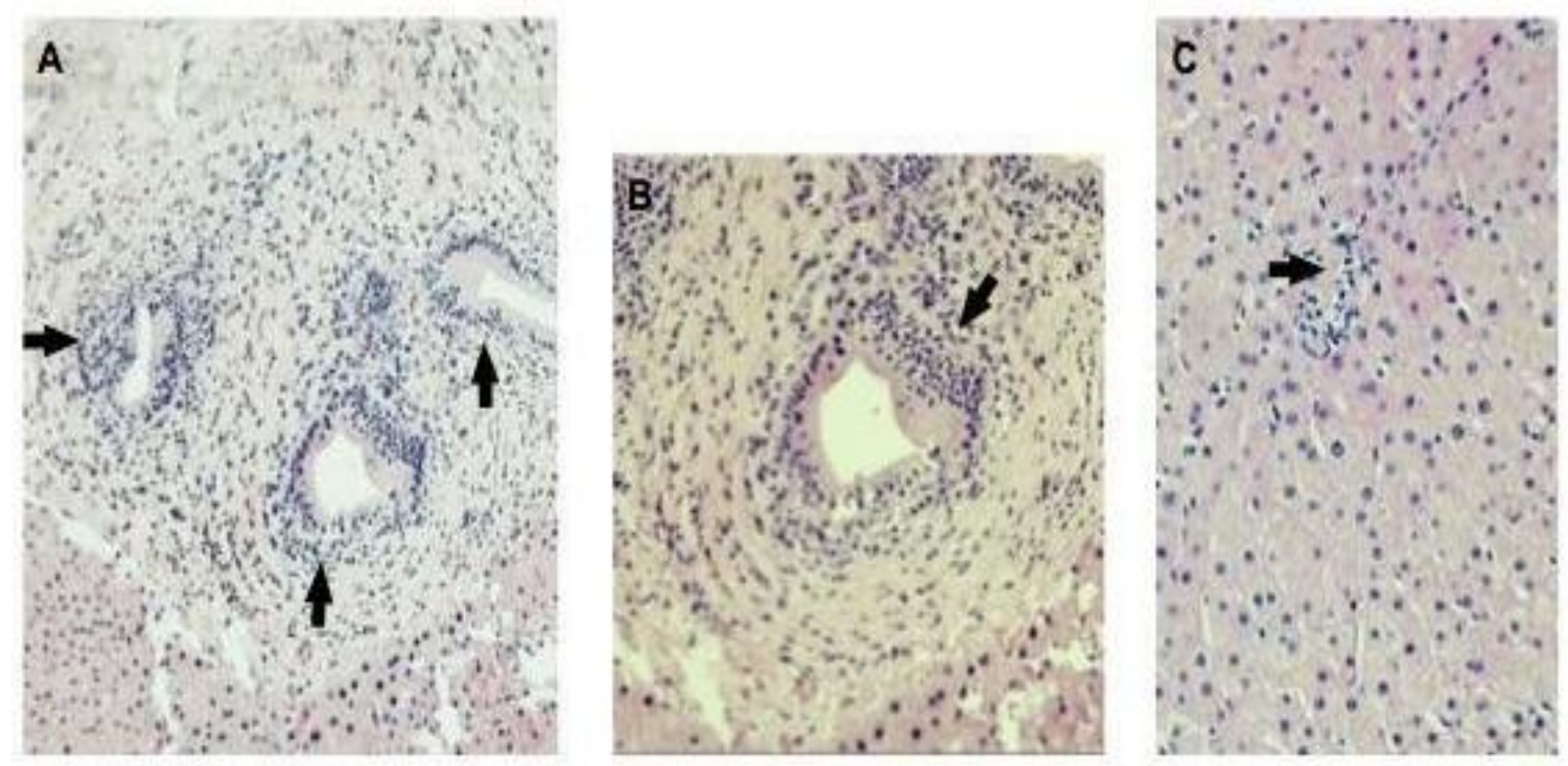

Legenda: A: Espaço porta septal com infiltrado e fibrose pericanalicular; B: Maior aumento do ducto septal circundado por fibrose e infiltrado inflamatório que penetra por vezes no epitélio glandular ocasionalmente vacuolado; C: Infiltrado sinusoidal leve.

Fonte: Mendes LSC, et al., 2021.

Foi realizado tratamento para CBP com Ácido Ursodesoxicólico $300 \mathrm{mg} 3 \mathrm{x} / \mathrm{dia}(15 \mathrm{mg} / \mathrm{kg} / \mathrm{dia})$. Paciente retorna em 2 meses, assintomática, TGO $29 \mathrm{U} / \mathrm{L}$; TGP $25 \mathrm{U} / \mathrm{L}$; FA $161 \mathrm{Ul} / \mathrm{ml}$; GGT $82 \mathrm{Ul} / \mathrm{ml}$; AMA 1:80; FAN negativo e colesterol em níveis normais sem uso adicional de outros hipolipemiantes e sem ajuste de dose. Após 12 meses de tratamento as bilirrubinas totais eram $0.42 \mathrm{mg} / \mathrm{dL}$ e eram as transaminases e FA dentro dos valores de referência (Figura 2). 
Figura 2 - Curva de transaminases após início do tratamento com ácido ursodesoxicólico.

- Transaminase Oxalacética TGO (AST)

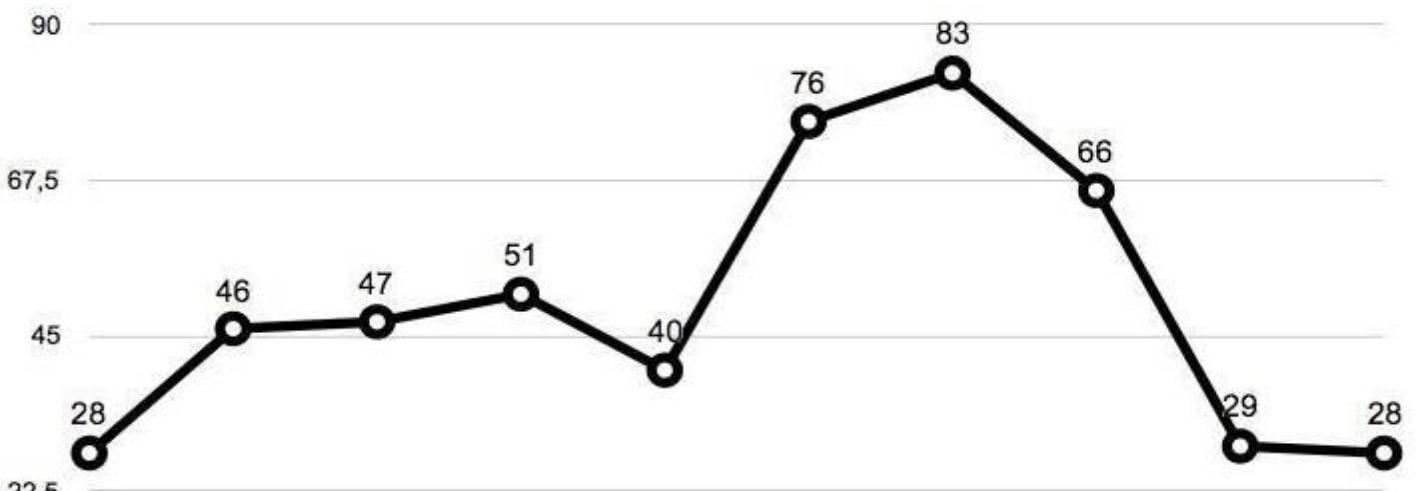

22,5

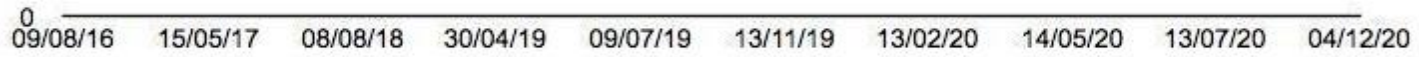

Método: IFCC sem piridoxal fosfato

Material: SANGUE

Valor de referência: menor que $34 \mathrm{U} / \mathrm{L}$

- Transaminase Pirúvica TGP (ALT)

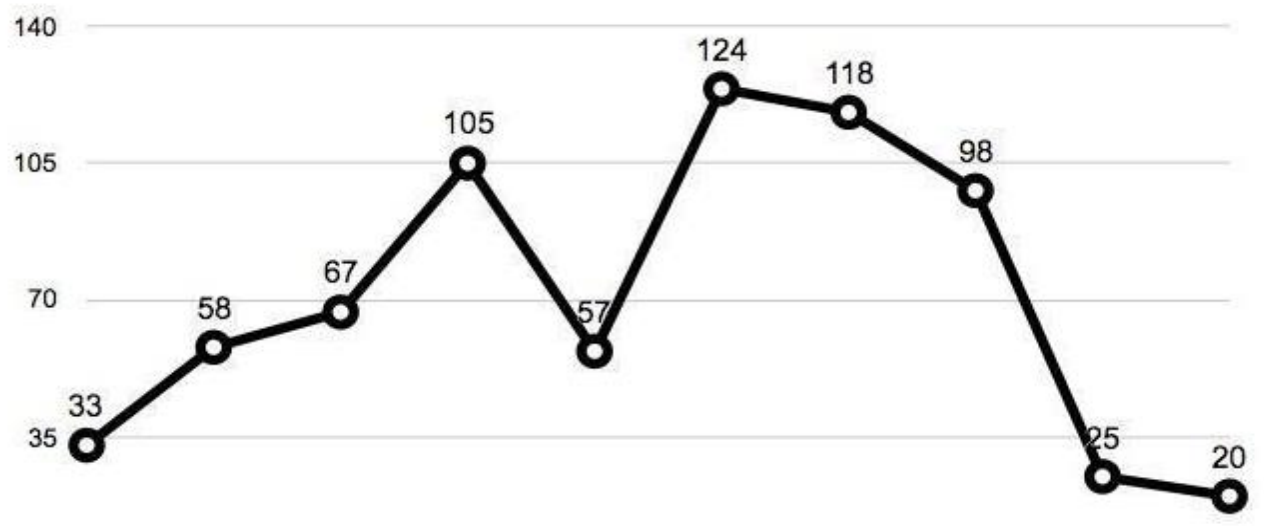

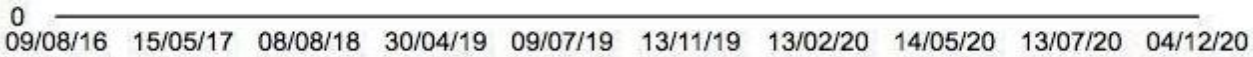

Método: IFCC sem piridoxal fosfato

Material: SANGUE

Valor de referência: 10 a $49 \mathrm{U} / \mathrm{L}$

Fonte: Mendes LSC, et al., 2021.

\section{DISCUSSÃO}

A Colangite Biliar Primária possui prevalência e incidência maiores em mulheres, nas proporções relativas de 3,9 e 3,2 respectivamente (LU M, et al., 2018) e acomete principalmente pacientes entre a quinta e sétima década de vida (IDILMAN IS, et al., 2020). Foi observado um aumento da prevalência de CPB entre os anos de 2006 e 2014, no entanto, sem o aumento concomitante da incidência de casos. Sugere-se então, que há aumento na expectativa de vida desses pacientes e/ou diagnóstico precoce (LU M, et al., 2018). Tal como foi visto no relato da paciente que tinha 55 anos quando foi diagnosticada com quadro de CBP, mas desde os 50 anos apresentava alterações persistentes das enzimas hepáticas. 
A clínica do paciente portador de CBP é caracterizada por uma variedade de sintomas associada a uma progressão arrastada. A maior parte dos pacientes são assintomáticos e o diagnóstico é feito a partir de exames de rotina da bioquímica hepática (KUMAGI T e HEATHCOTE E, et al., 2008).

Quando a doença se manifesta clinicamente os principais sintomas associados são a fadiga e prurido (LINDOR KD, et al., 2019). A fadiga foi encontrada em $50 \%$ a $78 \%$ dos pacientes e possui impacto negativo significativo na qualidade de vida (NEWTON JLJD, 2018). O prurido ocorre em $20 \%$ a $70 \%$ dos casos. Não há uma boa correlação entre esses sintomas e o estágio da doença, embora os pacientes com doença avançada geralmente apresentam mais sintomas (LINDOR KD, et al., 2019; PRINCE MI, et al., 2004).

A dor no quadrante superior direito é encontrada em aproximadamente 17\% dos pacientes com CBP, sendo tipicamente inespecífica em caráter, não progressiva, não bem correlacionada com o estágio da doença ou hepatomegalia e frequentemente desaparece espontaneamente (LAURIN JM, et al., 1994).

O exame físico do paciente portador de CBP é geralmente inocente, principalmente nos primeiros estágios da doença. Com a progressão desta, podem surgir sinais de hipertensão portal, como esplenomegalia e circulação venosa colateral (KUMAGI T e HEATHCOTE E, et al., 2008). A paciente em questão não relatava sintomas referentes à colestase crônica e também eram ausentes alterações compatíveis com colestase ao exame físico.

A tríade diagnóstica clássica é composta por (1) elevação de enzimas colestáticas, especialmente da (FA), em ao menos 1,5 vezes acima do normal (2) presença dos autoanticorpos AMA e por anticorpo antinuclear (FAN), por imunoflourescencia indireta, padrões nucleares tipo membrana nuclear e nuclear pontilhado com pontos isolados ou por Elisa, anti- gp210 e anti-sp-10 e (3) evidência histopatológica de colangite crônica não supurativa e destrutiva (KUMAGI T e HEATHCOTE E, et al., 2008). O diagnóstico clínico é presumível na presença de dois desses critérios. Métodos de imagem, como a colangiografia, associada a avaliação histológica podem auxiliar no diagnóstico em casos que o AMA é negativo (PRINCE MI, et al., 2003).

O curso da CBP é descrita inicialmente por níveis elevados de FA ( $\geq 1,5$ vezes o limite superior da normalidade) e GGT, ao passo que mais tardiamente há aumento dos níveis séricos de bilirrubina e diminuição da albumina sérica (HIRSCHFIELD GM, et al., 2018; SCTIE, 2021). O valor da FA tende a atingir um platô no início do curso da doença e, em seguida, geralmente flutua dentro de 20 por cento desse valor (CHRISTENSEN E, et al., 1980).

Aspartatoaminotransferase (AST/TGO) e alanino-aminotransferase (ALT/TGP) estão aumentadas em geral três vezes o limite superior normal (BOYER TD, et al., 2006). É raro que AST e ALT aumentem mais de cinco vezes o limite superior do normal, sugerindo que hepatite autoimune e CBP se sobrepõem ou coexiste hepatite viral (QAYED E, et al., 2016). O colesterol sérico eleva-se em pelo menos 50\% dos pacientes, podendo alcançar níveis de $1000 \mathrm{mg} / \mathrm{dL}$ em pacientes com xantelasma (FBG). No caso apresentado, o colesterol teve seus níveis reduzidos com tratamento específico para a CBP.

As alterações histopatológicas típicas da CBP é a colangite crônica não supurativa e destrutiva, que envolve ductos biliares interlobulares e está associada à infiltração inflamatória da veia porta com redução do ducto biliar (SELMI C, et al., 2011). A CBP tem quatro estágios histológicos: (1) a inflamação é limitada à tríade portal; (2) a inflamação se estende além da veia porta até o parênquima intralobular, e o número de ductos biliares normais diminui; (3) septos fibrosos ligando tratos portais; e (4) cirrose hepática (LLEO A, et al., 2003). As alterações histopatológicas do caso relatado são sugestivas de CBP em estadio 1, de acordo com a classificação de Peter Scheuer.

O tratamento mais empregado para a CBP é com ácido ursodesoxicólico (UDCA). Esta droga é um ácido biliar hidrofílico natural e possui mecanismos menos hepatotóxicos do que os ácidos biliares endógenos. Sua farmacodinâmica ainda não é completamente conhecida, mas sabe-se que ele inibe a síntese hepática do colesterol e estimula a síntese de ácidos biliares, restabelecendo o equilíbrio entre eles (SILVEIRA MG e LINDOR KD, 2019; SBH, 2015). Assim, o UDCA possui características citoprotetoras, coleréticas, imunomoduladoras, anti-inflamatórias e baixa propriedade hepatotóxica (BRASIL, 2019). 
Fatores de pior prognóstico incluem presença de sintomas no momento do diagnóstico, níveis elevados de fosfatase alcalina e bilirrubina, estágio histológico mais avançado, presença de anticorpos antinucleares, tabagismo e certos polimorfismos genético (MURILLO P, et al., 2020). No entanto, o prognóstico da doença melhorou acentuadamente com uso do UDCA. Pacientes em estágio inicial e com boa resposta bioquímica ao tratamento podem apresentar expectativa de vida igual a da população em geral (KUIPER EM, et al., 2009). A dose preconizada para a paciente foi de $15 \mathrm{mg} / \mathrm{kg}$ ao dia.

Modelos preditivos baseados em dados laboratoriais e clínicos foram propostos, e dois desses modelos (pontuação GLOBE e pontuação UK-PBC) foram desenvolvidos na era da terapia UDCA são baseados em estudos multicêntricos, incluindo grandes coortes de pacientes com CBP. O escore GLOBE estima a duração da sobrevida livre de transplante e inclui as seguintes cinco variáveis: bilirrubina sérica, albumina, fosfatase alcalina, contagem de plaquetas após um ano de tratamento com UDCA e idade no início da terapia (LAMMERS WJ, et al., 2015).

A chamada "resposta ao tratamento" prediz fortemente o resultado a longo prazo na colangite biliar primária (LINDOR KD, et al., 2019). Vários modelos de prognóstico de longo prazo baseados exclusivamente na resposta ao tratamento foram desenvolvidos e são amplamente usados para estratificar o risco de pacientes com CBP e orientar seu tratamento, como demonstrado na Tabela 1. No caso descrito, após 12 meses, estavam normais a FA, bilirrubinas e transaminases, perfazendo padrão de resposta por critério de PARIS I. No entanto, não levam em conta outras variáveis prognósticas, como o estágio da doença hepática.

Carbone M, et al. (2015) conduziu um estudo de coorte com 1249 pacientes tratados com UDCA validando a pontuação UK-PBC. Este modelo estima o risco de transplante hepático ou morte relacionada a doenças hepáticas e inclui como preditores a fosfatase alcalina sérica, aminotransferases e bilirrubina após 12 meses de terapia com UDCA. Além disso, inclui como preditores a albumina basal e a contagem de plaquetas. Estes critérios de resposta estão exemplificados na Tabela 1.

Tabela 1 - Critérios de resposta ao tratamento com Ácido Ursodesoxicólico e suas variáveis.

\begin{tabular}{ccc}
\hline Sistemas binários de resposta & Tempo (meses) & Falha ao tratamento \\
\hline Barcelona & 12 & Redução $<40 \%$ na FA e FA $\geq 1 \times$ LSN \\
\hline Paris I & 12 & FA $\geq 3 \times$ LSN ou AST $\geq 2 \times$ LSN ou bilirrubina $>$ \\
$1 \mathrm{~m} / \mathrm{dl}$
\end{tabular}

Legenda: GGT: gama-glutamiltransferase; FA: fosfatase alcalina; AST/TGO: aspartato aminotransferase; LSN: limite superior do normal

Fonte: Mendes LSC, et al., 2021; dados extraídos de Comissão Nacional de Incorporação de Tecnologias no SUS (CONITEC), 2018.

Cerca de $30 \%$ dos pacientes têm resposta completa ao tratamento com Ácido Ursodesoxicólico, com normalização bioquímica e melhora ou estabilização das lesões histológicas (FBG), como é demonstrado no caso clínico apresentado.

Desde 2016 foi aprovado o ácido obeticólico pelo Food and Drug Administration (FDA) e, desde então, vem se mostrando benéfico em associação ao AUDC nos não respondedores ao AUDC para tratamento de CBP (LINDOR KD, 2019). 
A CBP é uma doença rara, mal diagnosticada e com perspectiva de tratamento capaz de definir a melhora bioquímica e sobrevida livre de transplante. A análise do relato de caso de CBP e suas complicações é fundamental para que seja possível o aprimoramento das estratégias de diagnóstico e tratamento, possibilitando uma melhora na qualidade de vida destes pacientes. Por fim, o diagnóstico e tratamento precoces como visto no caso são fundamentais para evitar desfechos desfavoráveis.

\section{REFERÊNCIAS}

1. BEUERS U, et al. Changing nomenclature for PBC: From "Cirrhosis" to "Cholangitis". American Journal of Gastroenterology, 2015; 110(11).

2. BOYER TD, et al. Zakim and Boyer's Hepatology. $5^{\circ}$ Edição. Elsevier, 2006; 1584.

3. BRASIL. Ministério da Saúde. Portaria conjunta N 11, DE 09 DE SETEMBRO DE 2019.

4. BRASIL. Gastroenterologia, Sociedade Brasileira de Hepatologia. Cirrose biliar primária., 2008.

5. CARBONE M, et al. The UK-PBC risk scores: Derivation and validation of a scoring system for long-term prediction of end-stage liver disease in primary biliary cholangitis. Hepatology,2016; 3(63).

6. CHRISTENSEN E, et al. Clinical pattern and course of disease in primary biliary cirrhosis based on an analysis of 236 patients. Gastroenterology, 1980; 78(2).

7. GERSHWIN ME, et al. Identification and specificity of a cDNA encoding the $70 \mathrm{kd}$ mitochondrial antigen recognized in primary biliary cirrhosis. Journal of immunology (Baltimore, Md.: 1950), 1987; 138(10).

8. HARMS MH, et al. Ursodeoxycholic acid therapy and liver transplant-free survival in patients with primary biliary cholangitis. J Hepatol., 2019; 71(2): 357-365.

9. HIRSCHFIELD GM, et al. The British Society of Gastroenterology/UK-PBC primary biliary cholangitis treatment and management guidelines Gut, 2018.

10. IDILMAN IS, et al. Magnetic resonance imaging features in 283 patients with primary biliary cholangitis. European Radiology, 2020; 30(9).

11. JACOBSON BC. Sleisenger \& Fordtran's gastrointestinal and liver disease review and assessment to accompany Sleisenger \& Fordtran's Gastrointestinal and Liver Disease. Gastroenterology, 1999; 116(5).

12. KUIPER EM, et al. Improved Prognosis of Patients With Primary Biliary Cirrhosis That Have a Biochemical Response to Ursodeoxycholic Acid. Gastroenterology, 2009; 136(4).

13. KUMAGI T, HEATHCOTE E. Primary biliary cirrhosis. Orphanet Journal of Rare Diseases, 2008; 3(1).

14. LAMMERS WJ, et al. Development and Validation of a Scoring System to Predict Outcomes of Patients with Primary Biliary Cirrhosis Receiving Ursodeoxycholic Acid Therapy. Gastroenterology, 2015; 149(7).

15. LAURIN JM, et al. The Natural History of Abdominal Pain Associated with Primary Biliary Cirrhosis. The American Journal of Gastroenterology, 1984; 89(10).

16. LINDOR KD, et al. Primary Biliary Cholangitis: 2018 Practice Guidance from the American Association for the Study of Liver Diseases. Hepatology, 2019; 69(1).

17. LLEO A, et al. Etiopathogenesis of primary biliary cirrhosis World Journal of Gastroenterology, 2008.

18. LU M, et al. Increasing Prevalence of Primary Biliary Cholangitis and Reduced Mortality with Treatment. Clinical Gastroenterology and Hepatology, 2018; 16(8).

19. NEWTON JL, et al. Fatigue in primary biliary cirrhosis is associated with excessive daytime somnolence. Hepatology, 2006; 44(1).

20. PANDIT S, SAMANT H. Primary Biliary Cholangiti, 2021.

21. PEREZ CFM, et al. Goals of Treatment for Improved Survival in Primary Biliary Cholangitis: Treatment Target Should Be Bilirubin within the Normal Range and Normalization of Alkaline Phosphatase. American Journal of Gastroenterology, 2020; 115(7).

22. PRINCE MI, et al. Asymptomatic primary biliary cirrhosis: Clinical features, prognosis, and symptom progression in a large population based cohort Gut, 2004.

23. PRINCE MI, JAMES OFW. The epidemiology of primary biliary cirrhosis. Clinics in Liver Disease, 2003; 7(4).

24. SEMI C, et al. Primary biliary cirrhosis. The Lancet, 2011; 377(9777).

25.SILVA AEB, et al. Recomendações da Sociedade Brasileira de Hepatologia para Diagnóstico e Tratamento das Doenças Colestáticas e Hepatite Autoimune, 2014; 1-20. 